Appendix 1. Study design. *Eligible, consenting women on placebo during the preceding double-blind, placebocontrolled studies enrolled and were randomized to one of the two elagolix doses in the extension studies and received up to 6 months of elagolix treatment; data from these women will be reported in a separate publication.

Preceding Double-blind Studies

\section{Elaris EM-I}

Elaris EM-II

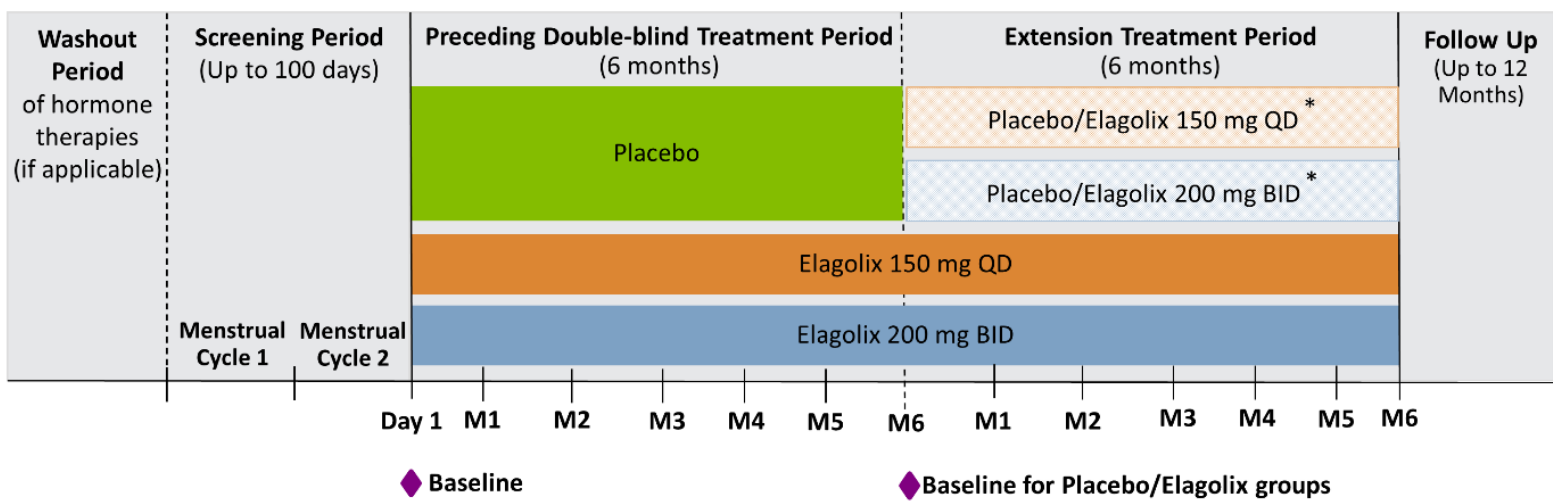

Surrey E, Taylor HS, Giudice L, Lessey BA, Abrao M, Archer DF, et al. Long-term outcomes of elagolix in women with endometriosis: results from two extension studies. Obstet Gynecol 2018; 132.

The authors provided this information as a supplement to their article. 
Appendix 2. Other Efficacy Measures after 12 Months of Treatment: Patient Global Impression of Change Scores, Changes in Analgesic Use, and Numeric Rating Scale Scores

\begin{tabular}{|c|c|c|c|c|}
\hline \multirow[b]{2}{*}{ Secondary Efficacy Outcome } & \multicolumn{2}{|c|}{ Elaris EM-III } & \multicolumn{2}{|c|}{ Elaris EM-IV } \\
\hline & $\begin{array}{l}\text { Elagolix } \\
\text { 150mg QD }\end{array}$ & $\begin{array}{c}\text { Elagolix } \\
\text { 200mg BID }\end{array}$ & $\begin{array}{l}\text { Elagolix } \\
\text { 150mg QD }\end{array}$ & $\begin{array}{l}\text { Elagolix } \\
\text { 200mg BID }\end{array}$ \\
\hline $\begin{array}{l}\text { Change in pill count from baseline, LS mean } \\
\pm \mathrm{SE}\end{array}$ & $N=117$ & $N=110$ & $N=122$ & $N=116$ \\
\hline Any Analgesic Use & $-0.40 \pm 0.70$ & $-0.56 \pm 0.67$ & $-0.45 \pm 0.83$ & $-0.59 \pm 0.80$ \\
\hline NSAID Only & $-0.27 \pm 0.52$ & $-0.31 \pm 0.43$ & $-0.26 \pm 0.57$ & $-0.32 \pm 0.38$ \\
\hline Opioid Only & $-0.13 \pm 0.44$ & $-0.25 \pm 0.53$ & $-0.20 \pm 0.62$ & $-0.27 \pm 0.68$ \\
\hline $\begin{array}{l}\text { Change in overall endometriosis-associated } \\
\text { pain from baseline*, LS mean score } \pm \text { SE }\end{array}$ & $-2.58 \pm 2.27$ & $-3.09 \pm 2.03$ & $-2.81 \pm 2.06$ & $-3.14 \pm 1.93$ \\
\hline $\begin{array}{l}\mathrm{n} / \mathrm{N}(\%) \text { with Much or Very Much Improved } \\
\text { Responses on PGIC }\end{array}$ & $77 / 111(69.4)$ & $93 / 102(91.2)$ & $86 / 114(75.4)$ & $89 / 106(84.0)$ \\
\hline
\end{tabular}

Statistical comparisons between treatment groups were not predefined and not performed. Analgesic use and pain scores based on average over 35-day window. *Measured with the Numeric Rating Scale; women provided daily self-assessments of overall endometriosis-associated pain on a scale of 0 (no pain) to 10 (worst pain ever). QD = once daily, BID = twice daily, PGIC = Patient Global Impression of Change, LS = least-squares, SE = standard error of the mean, NSAID = nonsteroidal anti-inflammatory drugs 
Appendix 3. Mean Endometriosis Health Profile-30 scores before and after 12 months of elagolix treatment. Baseline was prior to first treatment in the preceding double-blind, placebo-controlled trials. Statistical comparisons between treatment groups were not predefined and not performed. QD, once daily; BID, twice daily.
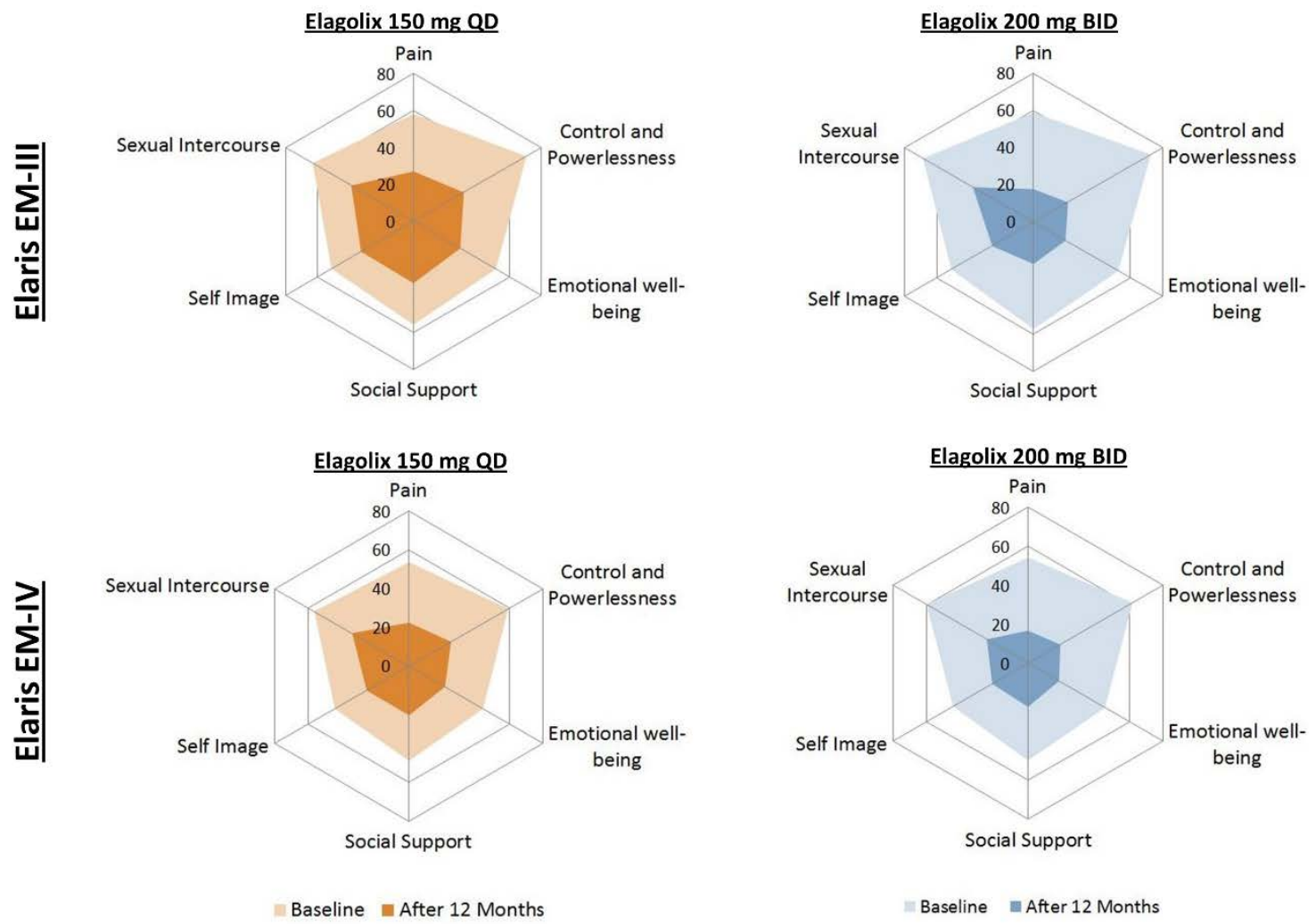

Surrey E, Taylor HS, Giudice L, Lessey BA, Abrao M, Archer DF, et al. Long-term outcomes of elagolix in women with endometriosis: results from two extension studies. Obstet Gynecol 2018; 132.

The authors provided this information as a supplement to their article. 
Appendix 4. Adverse Events Occurring in $\geq 5 \%$ of Women in Each Study Over the Course of 12 Months of Elagolix*

\begin{tabular}{|c|c|c|c|c|}
\hline \multirow[b]{3}{*}{$\mathrm{n}(\%)$} & \multicolumn{2}{|c|}{ Elaris EM-III } & \multicolumn{2}{|c|}{ Elaris EM-IV } \\
\hline & $\begin{array}{c}\text { Elagolix } \\
150 \mathrm{mg} \text { QD }\end{array}$ & $\begin{array}{c}\text { Elagolix } \\
\text { 200mg BID }\end{array}$ & $\begin{array}{c}\text { Elagolix } \\
150 \mathrm{mg} \text { QD }\end{array}$ & $\begin{array}{c}\text { Elagolix } \\
\text { 200mg BID }\end{array}$ \\
\hline & $\mathrm{N}=149$ & $\mathrm{~N}=138$ & $\mathrm{~N}=142$ & $\mathrm{~N}=140$ \\
\hline Hot flush & $44(29.5)$ & $72(52.2)$ & $36(25.4)$ & $77(55)$ \\
\hline Headache & $29(19.5)$ & $35(25.4)$ & $31(21.8)$ & $41(29.3)$ \\
\hline Nausea & $18(12.1)$ & $34(24.6)$ & $25(17.6)$ & $21(15)$ \\
\hline Urinary tract infection & $26(17.4)$ & $16(11.6)$ & $15(10.6)$ & $19(13.6)$ \\
\hline Sinusitis & $18(12.1)$ & $18(13)$ & $11(7.7)$ & $16(11.4)$ \\
\hline Upper respiratory tract infection & $25(16.8)$ & $10(7.2)$ & $11(7.7)$ & $14(10)$ \\
\hline Fatigue & $19(12.8)$ & $13(9.4)$ & $6(4.2)$ & $8(5.7)$ \\
\hline Nasopharyngitis & $15(10.1)$ & $16(11.6)$ & $16(11.3)$ & $22(15.7)$ \\
\hline Insomnia & $12(8.1)$ & $18(13)$ & $14(9.9)$ & $15(10.7)$ \\
\hline Back Pain & $11(7.4)$ & $17(12.3)$ & $11(7.7)$ & $21(15.0)$ \\
\hline Abdominal pain & $11(7.4)$ & $13(9.4)$ & $9(6.3)$ & $8(5.7)$ \\
\hline Anxiety & $11(7.4)$ & $13(9.4)$ & $8(5.6)^{\dagger}$ & $5(3.6)^{\dagger}$ \\
\hline Vomiting & $13(8.7)$ & $10(7.2)$ & $7(4.9)$ & $9(6.4)$ \\
\hline Depression & $13(8.7)$ & $9(6.5)$ & $3(2.1)^{\dagger}$ & $3(2.1)^{\dagger}$ \\
\hline Influenza & $10(6.7)$ & $10(7.2)$ & $6(4.2)+$ & $7(5.0)^{\dagger}$ \\
\hline Bronchitis & $11(7.4)$ & $9(6.5)$ & $7(4.9)$ & $8(5.7)$ \\
\hline Diarrhea & $7(4.7)$ & $12(8.7)$ & $12(8.5)$ & $10(7.1)$ \\
\hline Mood swings & $4(2.7)$ & $14(10.1)$ & $12(8.5)$ & $6(4.3)$ \\
\hline Gastroenteritis viral & $6(4)$ & $12(8.7)$ & $5(3.5) \dagger$ & $1(0.7)+$ \\
\hline Arthralgia & $7(4.7)$ & $11(8)$ & $13(9.2)$ & $18(12.9)$ \\
\hline Vulvovaginal mycotic infection & $10(6.7)$ & $8(5.8)$ & $4(2.8)^{\dagger}$ & $2(1.4)+$ \\
\hline Acne & $9(6)$ & $8(5.8)$ & $6(4.2)$ & $9(6.4)$ \\
\hline Night sweats & $6(4)$ & $10(7.2)$ & $1(0.7)^{\dagger}$ & $4(2.9) \dagger$ \\
\hline Dizziness & $8(5.4)$ & $8(5.8)$ & $10(7.0)+$ & $3(2.1) \dagger$ \\
\hline Amenorrhea & $4(2.7)$ & $11(8)$ & $8(5.6)$ & $17(12.1)$ \\
\hline C-reactive protein increased & $6(4)$ & $9(6.5)$ & $5(3.5)^{\dagger}$ & $4(2.9) \dagger$ \\
\hline Migraine & $6(4)$ & $9(6.5)$ & $2(1.4)^{\dagger}$ & $2(2.9) \dagger$ \\
\hline Weight Increased & $8(5.4) \ddagger$ & $6(4.3) \neq$ & $9(6.3)$ & $8(5.7)$ \\
\hline Constipation & $8(5.4) \ddagger$ & $6(4.3) \ddagger$ & $7(4.9)$ & $7(5.0)$ \\
\hline Pelvic Pain & $6(4.0) \ddagger$ & $7(5.1) \ddagger$ & $7(4.9)$ & $7(5.0)$ \\
\hline
\end{tabular}

*In descending order of GnRH antagonist treatment overall in Elaris EM-III, then Elaris EM-IV; only women that received GnRH antagonist during the preceding double-blind, placebo-controlled trials, IIncidence not $\geq 5 \%$ overall in Elaris EM-IV, $¥$ Incidence not $\geq 5 \%$ overall in Elaris EM-III. QD = once daily, BID = twice daily.

Surrey E, Taylor HS, Giudice L, Lessey BA, Abrao M, Archer DF, et al. Long-term outcomes of elagolix in women with endometriosis: results from two extension studies. Obstet Gynecol 2018; 132.

The authors provided this information as a supplement to their article.

C 2018 American College of Obstetricians and Gynecologists. 


\section{Appendix 5. Summary of Adverse Events With New Onset in Each Extension Study}

\begin{tabular}{|c|c|c|c|c|}
\hline \multirow[b]{3}{*}{ n (\%) } & \multicolumn{2}{|c|}{ Elaris EM-III } & \multicolumn{2}{|c|}{ Elaris EM-IV } \\
\hline & $\begin{array}{l}\text { Elagolix } \\
\text { 150mg QD }\end{array}$ & $\begin{array}{l}\text { Elagolix } \\
\text { 200mg BID }\end{array}$ & $\begin{array}{l}\text { Elagolix } \\
\text { 150mg QD }\end{array}$ & $\begin{array}{l}\text { Elagolix } \\
\text { 200mg BID }\end{array}$ \\
\hline & $\mathrm{N}=149$ & $\mathrm{~N}=138$ & $\mathrm{~N}=142$ & $\mathrm{~N}=140$ \\
\hline Any adverse event $(\mathrm{AE})$ & $111(74)$ & $102(74)$ & $116(81.7)$ & $109(77.9)$ \\
\hline Any serious $A E$ & $5(3.4)$ & $4(2.9)$ & $7(4.9)$ & $8(5.7)$ \\
\hline Any severe $A E$ & $18(12)$ & $22(16)$ & $9(6.3)$ & $11(7.9)$ \\
\hline Any AE leading to discontinuation & $6(4.0)$ & $12(8.7)$ & $8(5.6)$ & $10(7.1)$ \\
\hline Deaths & 0 & 0 & 0 & 0 \\
\hline \multicolumn{5}{|l|}{$\begin{array}{l}\text { AEs occurring in } \geq 4 \% \text { of women overall in } \\
\text { each study* } n(\%)\end{array}$} \\
\hline Urinary Tract Infection & $17(11.4)$ & $11(8.0)$ & $10(7.0)$ & $11(7.9)$ \\
\hline Sinusitis & $10(6.7)$ & $10(7.2)$ & $8(5.6)$ & $8(5.7)$ \\
\hline Upper Respiratory Tract Infection & $16(11)$ & $4(2.9)$ & $6(4.2)$ & $6(4.3)$ \\
\hline Headache & $10(6.7)$ & $8(5.8)$ & $9(6.3)$ & $9(6.4)$ \\
\hline Nasopharyngitis & $9(6.0)$ & $9(6.5)$ & $6(4.2)$ & $9(6.4)$ \\
\hline Nausea & $7(4.7)$ & $10(7.2)$ & $14(9.9)$ & $5(3.6)$ \\
\hline Back Pain & $6(4.0)$ & $10(7.2)$ & $6(4.2)$ & $10(7.1)$ \\
\hline Vulvovaginal Mycotic Infection & $8(5.4)$ & $6(4.3)$ & $1(0.7)+$ & $1(0.7)+$ \\
\hline Abdominal Pain & $7(4.7)$ & $8(5.8)$ & $4(2.8)^{\dagger}$ & $6(4.3) \dagger$ \\
\hline Bronchitis & $8(5.4)$ & $7(5.1)$ & $4(2.8)^{\dagger}$ & $2(1.4)^{\dagger}$ \\
\hline Fatigue & $9(6.0)$ & $5(3.6)$ & $0+$ & $2(1.4)^{\dagger}$ \\
\hline Hot Flush & $6(4.0)$ & $8(5.8)$ & $7(4.9)$ & $11(7.9)$ \\
\hline Depression & $8(5.4)$ & $4(2.9)$ & $1(0.7)^{\dagger}$ & $0+$ \\
\hline Influenza & $6(4.0)$ & $6(4.3)$ & $3(2.1)^{\dagger}$ & $4(2.9) \dagger$ \\
\hline Vomiting & $6(4.0)$ & $6(4.3)$ & $4(2.8)^{\dagger}$ & $4(2.9)^{\dagger}$ \\
\hline Arthralgia & $2(1.3) \ddagger$ & $7(5.1) \ddagger$ & $6(4.2)$ & $9(6.4)$ \\
\hline Diarrhea & $2(1.3) \ddagger$ & $5(3.6) \ddagger$ & $6(4.2)$ & $6(4.3)$ \\
\hline
\end{tabular}

*In descending order of elagolix overall in Elaris EM-III, then Elaris EM-IV; only women that received elagolix during the preceding double-blind, placebo-controlled trials, Incidence not $\geq 4 \%$ overall in Elaris EM-IV, $\ddagger$ Incidence not $\geq$ $4 \%$ overall in Elaris EM-III. QD = once daily, $\mathrm{BID}=$ twice daily, $\mathrm{AE}=$ adverse event.

Surrey E, Taylor HS, Giudice L, Lessey BA, Abrao M, Archer DF, et al. Long-term outcomes of elagolix in women with endometriosis: results from two extension studies. Obstet Gynecol 2018; 132.

The authors provided this information as a supplement to their article. 
Appendix 6. Median, quartile 1, quartile 3, minimum and maximum of bone mineral density Z-scores over the course of 12 months of elagolix treatment (Elaris EM-III and IV) and at post-treatment months 6 and 12* (Elaris EM-IV only). Baseline was prior to dosing in the preceding double-blind, placebo-controlled trials (Elaris EM-I and Elaris EM-II). Dotted lines indicate the normal age- and race-matched range. Month 12 during the extension treatment period includes women who prematurely discontinued and women that had $\geq 12$ months of elagolix treatment (as discussed in the methods). Error bars represent $95 \%$ confidence interval for mean percentage change from baseline. Statistical comparisons between treatment groups were not predefined and not performed. *Elaris EM-III was not designed to evaluate post-treatment bone mineral density recovery for all women. For Elaris EM-IV, all women were required to have a follow-up DXA at 6 months post-treatment. However only women who had a decrease $>1.5 \%$ from baseline in lumbar spine or a decrease $>2.5 \%$ in total hip BMD at 6 months post-treatment were required to have a follow-up DXA at 12 months post-treatment. QD, once daily; BID; twice daily; BL, baseline; Ext, extension; M, month; DXA, dual energy X-ray absorptiometry.
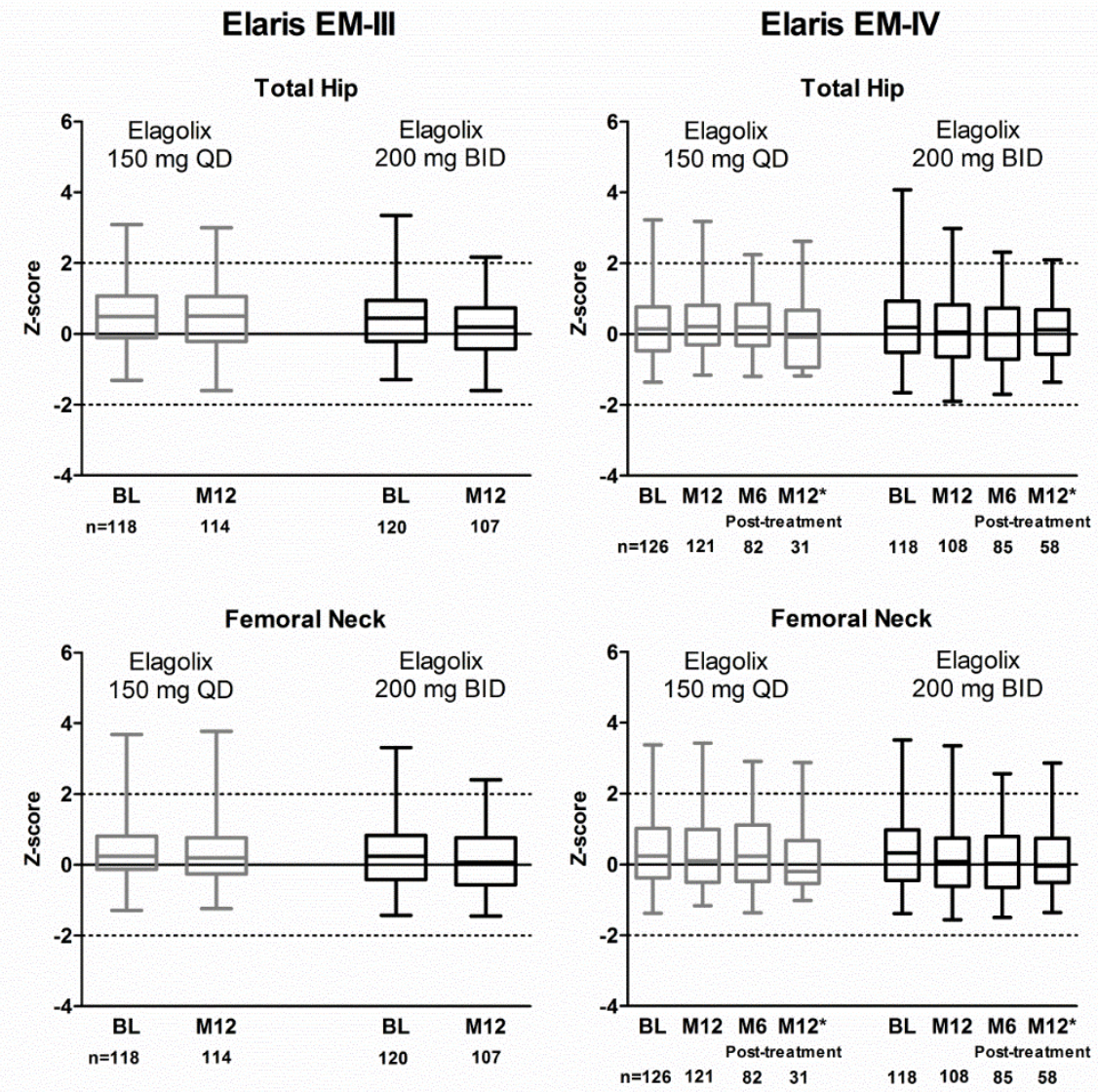

Surrey E, Taylor HS, Giudice L, Lessey BA, Abrao M, Archer DF, et al. Long-term outcomes of elagolix in women with endometriosis: results from two extension studies. Obstet Gynecol 2018; 132.

The authors provided this information as a supplement to their article. 
Appendix 7. Number and Percentage of Women with Amenorrhea at Each Month in the Extension Studies

\begin{tabular}{l|c|c|c|c}
\multirow{2}{*}{ Women with Amenorrhea, $\mathbf{n} / \mathbf{N}(\%)$} & \multicolumn{2}{|c}{ Elaris EM-III } & \multicolumn{2}{c}{ Elaris EM-IV } \\
\cline { 2 - 5 } & $\begin{array}{c}\text { Elagolix } \\
150 \mathrm{mg} \text { QD }\end{array}$ & $\begin{array}{c}\text { Elagolix } \\
\text { 200mg BID }\end{array}$ & $\begin{array}{c}\text { Elagolix } \\
\text { 150mg QD }\end{array}$ & $\begin{array}{c}\text { Elagolix } \\
200 \mathrm{mg} \text { BID }\end{array}$ \\
\hline Extension study month & & & & \\
\hline 1 & $33 / 146(23)$ & $88 / 136(65)$ & $33 / 140(24)$ & $95 / 139(68)$ \\
\hline 2 & $43 / 137(31)$ & $88 / 128(69)$ & $38 / 138(28)$ & $93 / 134(69)$ \\
\hline 3 & $41 / 131(31)$ & $80 / 123(65)$ & $34 / 134(25)$ & $87 / 127(69)$ \\
\hline 4 & $38 / 127(30)$ & $78 / 119(66)$ & $27 / 127(21)$ & $85 / 124(69)$ \\
\hline 5 & $36 / 124(29)$ & $72 / 114(63)$ & $32 / 125(26)$ & $74 / 118(63)$ \\
\hline 6 & $25 / 94(27)$ & $57 / 90(63)$ & $18 / 89(20)$ & $59 / 97(61)$ \\
\hline
\end{tabular}

Statistical comparisons between treatment groups were not predefined and not performed. *A woman was considered amenorrheic if she did not report menstrual period or any uterine bleeding in the e-Diary during each 28-day period of interest and answered in the e-Diary at least once. Days with missing electronic diary entries were considered 'no bleeding' days. QD = once daily, BID = twice daily. 